\title{
A Whole-Body Sensory-Motor Gradient is Revealed in the Medial Wall of the Parietal Lobe
}

\author{
- Noa Zeharia, ${ }^{1,2 *}$ Shir Hofstetter, ${ }^{1,2,3 *}$ Tamar Flash, ${ }^{4}$ and ${ }^{\circledR}$ Amir Amedi ${ }^{1,2}$ \\ ${ }^{1}$ The Edmond and Lily Safra Center for Brain Sciences and Medical Neurobiology Department of Institute for Medical Research Israel-Canada and The \\ Hebrew University of Jerusalem Medical School, 91220, ${ }^{2}$ Hebrew University of Jerusalem, Jerusalem 91220, Israel, ${ }^{3}$ Spinoza Centre for Neuroimaging, 1105 \\ BK Amsterdam, Netherlands, and ${ }^{4}$ Department of Computer Science and Applied Mathematics, Weizmann Institute of Science, Rehovot 76 100, Israel
}

In 1954, Penfield and Jasper's findings based on electric stimulation of epileptic patients led them to hypothesize that a sensory representation of the body should be found in the precuneus. They termed this representation the "supplementary sensory" area and emphasized that the exact form of this homunculus could not be specified on the basis of their results. In the decades that followed, their prediction was neglected. The precuneus was found to be involved in numerous motor, cognitive and visual processes, but no work was done on its somatotopic organization. Here, we used a periodic experimental design in which 16 human subjects (eight women) moved 20 body parts to investigate the possible body part topography of the precuneus. We found an anterior-to-posterior, dorsal-to-ventral, toes-to-tongue gradient in a mirror orientation to the SMA. When inspecting body-part-specific functional connectivity, we found differential connectivity patterns for the different body parts to the primary and secondary motor areas and parietal and visual areas, and a shared connectivity to the extrastriate body area, another topographically organized area. We suggest that a whole-body gradient can be found in the precuneus and is connected to multiple brain areas with different connectivity for different body parts. Its exact role and relations to the other known functions of the precuneus such as self-processing, motor imagery, reaching, visuomotor and other bodymind functions should be investigated.

Key words: connectivity; homunculus; somatotopic gradient

\section{Significance Statement}

Using fMRI, as well as sensitive spectral analysis, we found a new homunculus in the precuneus: an anterior-to-posterior, dorsalto-ventral, toes-to-tongue somatotopic gradient in a mirror orientation to the SMA. When inspecting body-part-specific functional connectivity, we found differential connectivity patterns for the different body parts to the primary and secondary motor areas, parietal and visual areas, and a shared connectivity to the extrastriate body area, another topographically organized area. We suggest that a whole-body gradient can be found in the precuneus and is connected to multiple brain areas in a body-partspecific manner.

\section{Introduction}

In their 1954 book (Penfield and Jasper, 1954), Penfield and Jasper summarized their extensive scientific investigations, which

Received March 20, 2018; revised July 23, 2019; accepted July 26, 2019.

Author contributions: N.Z., T.F., and A.A. designed research; N.Z., T.F., and A.A. performed research; N.Z., S.H., and T.F. analyzed data; N.Z., T.F., and A.A. wrote the first draft of the paper; N.Z., S.H., T.F., and A.A. edited the paper; N.Z., S.H., T.F., and A.A. wrote the paper; A.A. contributed unpublished reagents/analytic tools.

This work was supported by an the European Research Council (Consolidator Grant \#773121 to A.A.); the James S. McDonnell Foundation (Scholar Award \#220020284 to A.A.); Joy Ventures (Grant for Innovating Neuro-Wellness to A.A.); the Israeli Center of Research Excellence (I-CORE) in the Cognitive Sciences (Grant \#51/11h to T.F.); and the VERE project funded under the European Seventh Framework Program (Grant \#EC-ICT-257695 to T.F.).

The authors declare no competing financial interests.

*N.Z. and S.H. contributed equally to this work.

Correspondence should be addressed to Amir Amedi at Amir.Amedi@ekmd.huji.ac.il.

https://doi.org/10.1523/JNEUROSCI.0727-18.2019

Copyright $@ 2019$ the authors drew primarily on observations of the cortex of epileptic patients undergoing invasive electric stimulation.

Their work described the now well established somatotopic organization of M1 and S1 (Meier et al., 2008; Zeharia et al., 2012), and of the SMA (Chainay et al., 2004; Zeharia et al., 2012). However, another result fell by the wayside. This was Penfield's hypothesis that another sensory body representation that covered the legs, arms, and face was located in the parietal medial wall, in the precuneus (Penfield and Jasper, 1954). They termed this hypothesized homunculus the "supplementary sensory" area, similar to the "supplementary motor" area now known as the SMA.

The suggested location of this homunculus was therefore the medial parietal wall, and its orientation was depicted as a mirror orientation of the SMA, with the leg area of M1 separating these two homunculi. However, this assumption remained a hypothe- 
sis. Penfield and Jasper (1954) drew this homunculus using dashes rather than solid lines and stated: "In regard to the sensory figure, our observations are not yet numerous enough to justify conclusions as to form or exact position."

In the decades that followed but especially in recent years, extensive research has been conducted to elucidate the roles of the precuneus. One of the most influential of these studies was conducted by Margulies et al. (2009), in which patterns of functional connectivity from the precuneus were examined in both humans and macaque monkeys and compared with the known anatomical connections in the macaque. The results indicated that the precuneus can be divided into three parts in both humans and monkeys: the anterior part, which is functionally connected to the motor cortex, the insula, and the superior parietal lobe; the middle part, which is functionally connected to prefrontal lobe and to the inferior parietal lobe; and the posterior part, which is functionally connected to visual areas. Based on these observations, the authors also suggested that the anterior part is the sensory-motor part of the precuneus, the middle part is an associative-cognitive area, and the posterior part is a vision-related area.

However, other studies have found evidence for motor as well as visuomotor related activations of the hand, and specifically reaching, in different parts of the precuneus that are not confined to the anterior part (Connolly et al., 2003; Filimon et al., 2009; Cavina-Pratesi et al., 2010; Vesia et al., 2010; Konen et al., 2013; Pitzalis et al., 2013; Rossit et al., 2013). Some of these activations (Pitzalis et al., 2013) are even located in the most posterior part of the precuneus, near or in the anterior bank of the parietaloccipital sulcus (POS).

Given the ample evidence for relationships between reachingrelated activations and visual-related activations, Filimon et al. (2007) showed that the precuneus is activated by reaching, observation of reaching and imagination of reaching, which thus suggests that this area is a part of the reaching mirror system. Other studies also suggested that the precuneus plays a role in motor imagery, mainly of the hands (Hanakawa et al., 2003; Wenderoth et al., 2005), but also of the legs (Malouin et al., 2003), and is also involved in cognitive processes such as self-processing and episodic memory retrieval (for review, see Cavanna and Trimble, 2006).

Here, we investigated whether in addition to the growing recent evidence for reaching-related activations in various parts of the precuneus, there is also evidence for a whole-body, sensorymotor representation similar to the one hypothesized by Penfield and Jasper (1954), and comparable to the recently found wholebody tactile-somatosensory map at the lateral surface of the parietal lobe (Sereno and Huang, 2006; Huang et al., 2012; Sereno and Huang, 2014). We also investigated the relationships between sensory-motor activations in the precuneus, and the motor-cognitive-visual dissociation suggested by Margulies et al. (2009).

\section{Materials and Methods \\ Experimental design and statistical analysis \\ Subjects}

16 healthy, right-handed subjects (eight women) aged 25-35 participated in the periodic (11 subjects) and the slow-event-related (seven subjects) experiments. Two of the subjects participated in both the periodic and event-related experiments. The experimental procedure was approved by the local ethics committee and written informed consent was obtained from all subjects. The details of the periodic experiments and the eventrelated experiment are also described elsewhere and the same subjects and data were used here (Zeharia et al., 2012).

\section{Training and movement recording}

The subjects were trained for approximately an hour before entering the scanner. During training, their movements were recorded by the Polhemus LIBERTY 240/16 tracking device to ensure that they were able to perform the movements perfectly and that the only body parts moved were as per instructions. In two subjects, EMGs were recorded to ensure correct performance of the task.

\section{Periodic experiments}

Toes to tongue. Subjects moved 20 body parts consecutively while lying with their eyes closed and blindfolded inside the fMRI scanner. The movement sequence followed the order from toes to tongue described by Penfield and Boldrey, 1937) in the primary motor cortex homunculus. It included bilateral movements of the following body parts: toes (flexion/ extension), feet (flexion/extension), thighs (contraction), buttocks (contraction), stomach (contraction), upper arm (contraction), elbow (flexion/extension), wrist (flexion/extension), fist (contraction), little finger (flexion/extension), ring finger (flexion/extension), middle finger (flexion/extension), index finger (flexion/extension), thumb (flexion/extension), forehead (contraction), nose (contraction), eyelids (contraction), lips (contraction), jaw (flexion/extension), and tongue (a side to side movement with the mouth closed). The subjects were instructed to execute the movements on hearing an auditory cue, which was the spoken name of the body part followed by three metronome beeps at one second intervals, during which the subject moved the body part. Overall, each body part was moved 3 times within a period of $3 \mathrm{~s}$, and the next body part was announced during the last second. The whole movement cycle lasted $60 \mathrm{~s}$ and was followed by a rest period of $12 \mathrm{~s}$. Eight cycles of movement and rest were performed, at a stimulus frequency of $0.0138 \mathrm{~Hz}$.

Tongue to toes. Eight of the 11 subjects who participated in the "toes to tongue" experiment also participated in this experiment. This experiment was identical to the "toes to tongue" experiment but the movements were performed in the reverse order, from tongue to toes.

\section{Event-related experiment}

All 20 body parts in the periodic experiments were moved 20 times each, in an event-related paradigm and in a pseudorandomized order balanced for first order history. In each event, a $4.5 \mathrm{~s}$ block of movement was followed by a $12 \mathrm{~s}$ rest. At the beginning of each movement period, subjects heard the name of the body part to be moved. Movement was paced with a metronome, and five movements were performed in each event at a frequency of $1 \mathrm{~Hz}$. The 400 movement-rest periods were divided into 10 runs. After five runs, subjects left the scanner for $20 \mathrm{~min}$ and then returned for the remaining five runs.

\section{Functional MRI acquisition}

The BOLD fMRI measurements were obtained in a whole-body $3 \mathrm{~T}$ Magnetom Trio scanner, with 12 channels (Siemens). The fMRI protocols were based on multi-slice gradient echoplanar imaging (EPI) and a standard head coil. The functional data were collected under the following timing parameters: time to repetition $(\mathrm{TR})=1.5 \mathrm{~s}$, time to echo $(\mathrm{TE})=30 \mathrm{~ms}, \mathrm{FA}=70^{\circ}$, imaging matrix $=80 \times 80$, field of view $(\mathrm{FOV})=24 \times 24 \mathrm{~cm}$ (i.e., in-plane resolution of $3 \mathrm{~mm}$ ). We used a relatively short TR value to later superimpose the phase-locking spectral analysis approach. Twenty six slices with slice thickness $=4.5 \mathrm{~mm}$ and no gaps were oriented in the axial position for complete coverage of the cortex.

Anatomical MRI acquisition and cortical surface reconstruction Separate 3D recordings were used for co-registration. High-resolution $3 \mathrm{D}$ anatomical volumes were collected using T1-weighted images using a 3D-turbo field echo (TFE) T1-weighted sequence (equivalent to MPRAGE). Typical parameters were as follows: FOV $23 \mathrm{~cm}(\mathrm{RL}) \times 23 \mathrm{~cm}$ $(\mathrm{VD}) \times 17 \mathrm{~cm}(\mathrm{AP})$; foldover axis: $\mathrm{RL}$, data matrix: $160 \times 160 \times 144$ zero-filled to 256 in all directions ( $\sim 1 \mathrm{~mm}$ isovoxel native data), TR/ $\mathrm{TE}=9 \mathrm{~ms} / 6 \mathrm{~ms}$, flip angle $=8^{\circ}$. Group results were superimposed on a 3D cortical reconstruction of a Talairach normalized brain (Talairach and Tournoux, 1988). Cortical reconstruction included the segmentation of the white matter using a grow-region function embedded in the 
A SMA and a hypothesized parietal homunculus in the medial wall
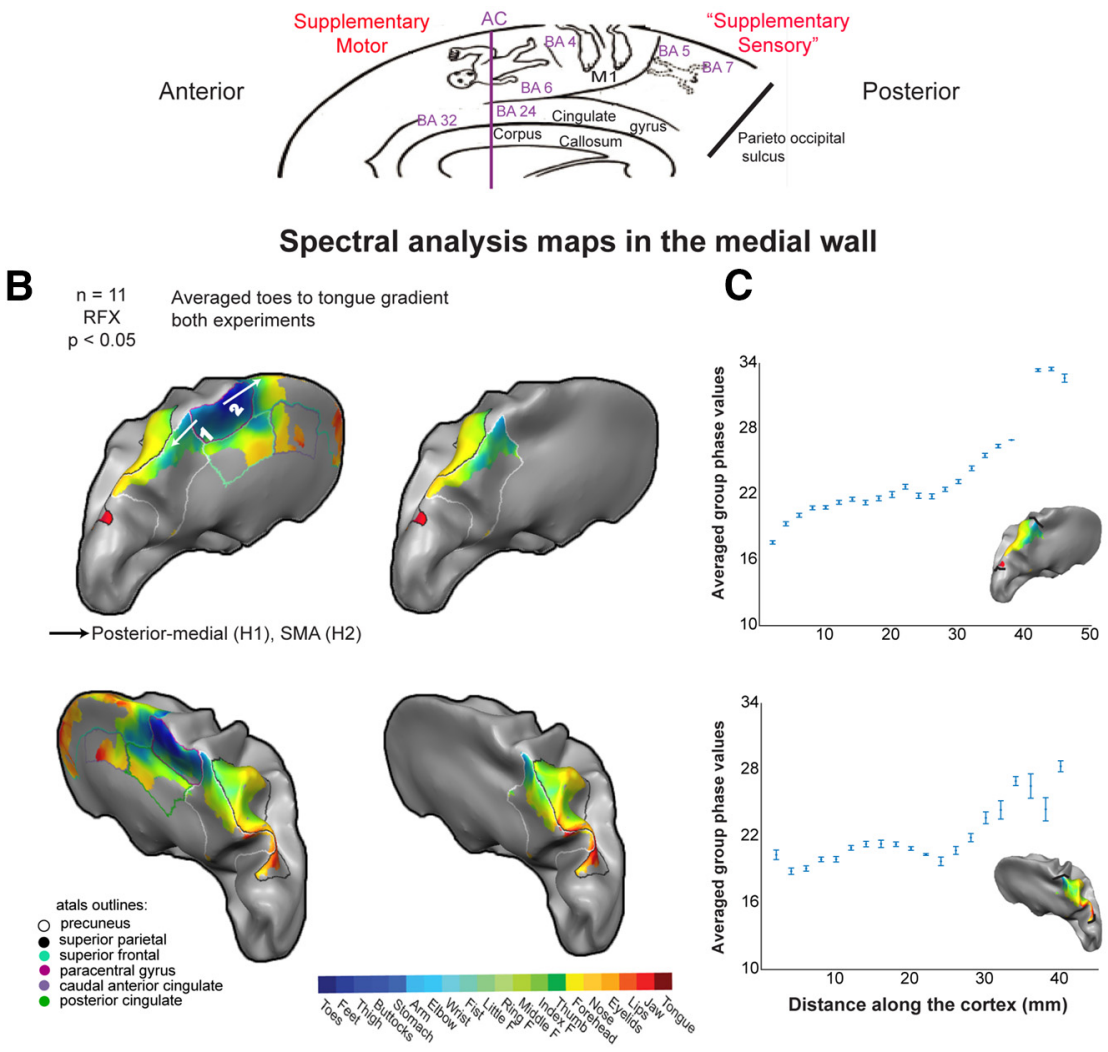

D

Spectral analysis maps the medial wall - Single subjects

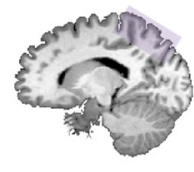

$\mathbf{E}$

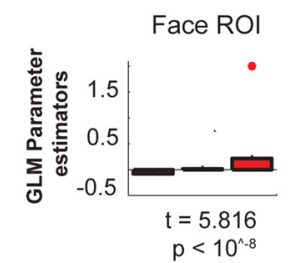

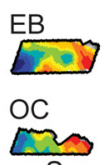

$\mathrm{S}$

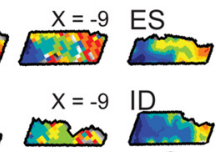

US

\section{ROI analysis}
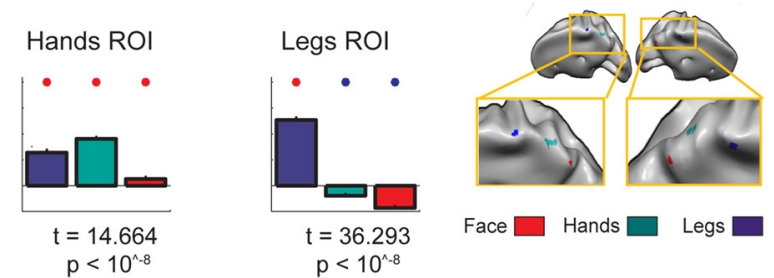

$\mathbf{F}$

SMA and a medial parietal homunculus - revised model

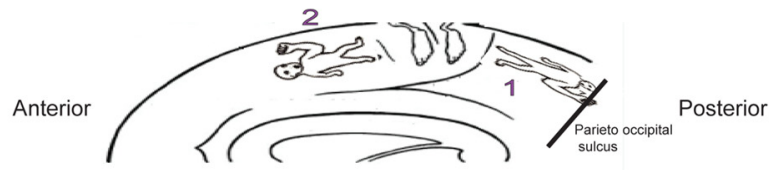

Figure 1. Spectral analysis reveals a somatotopic gradient in the precuneus. $\boldsymbol{A}$, Penfield and Jasper's prediction of a supplementary sensory area in the precuneus, posterior to the medial leg area of $\mathrm{M} 1$, in a mirror orientation to the SMA (reproduced from Penfield and Jasper, 1954). B. Group random effects (RFX) corrected for multiple-comparisons results of spectral analysis in the medial wall reveal an anterior-to-posterior, dorsal-to-ventral, toes-to-tongue gradient (1) in the precuneus, somewhat lateral to the midline, in a similar orientation to the one suggested in $\boldsymbol{A}$. The gradient in the SMA (2) is also shown, as well as the known gradients in the caudal cingulate zone and the rostral cingulate zone at the midline. The anatomical landmarks displayed on the surfaces were used to mask the whole-brain phase map (DKT classifier atlas; Desikan et al., 2006). The right maps present the new medial-posterior homunculi using anatomical masks of the precuneus and the superior parietal. C, Mean phase values progression across the medial-parietal maps. Voxels were organized by their distance from the black lines in the anterior-posterior borders of the group phase map. The phase value served as a measure of the body part being moved (see Materials and Methods). D, Spectral analysis results in the precuneus of four single subjects in the left and right hemispheres using spatially smoothed $(S)$ and
Brain Voyager QX 2.1.2 software package. The cortical surface was then inflated.

\section{Preprocessing}

The first 10 images (during the first baseline rest condition) were excluded from the analysis in all experiments because of non-steady-state magnetization. Data were mainly preprocessed using the Brain Voyager QX software package (Brain Innovation). fMRI data preprocessing included head motion correction. The maximum value of head movements in the periodic experiments was $2.46 \mathrm{~mm}$ (in the order of magnitude of one functional voxel; i.e., relatively negligible head motion), and the mean was $0.21 \pm 0.28 \mathrm{~mm}$. Slice scan time correction and high-pass filtering using temporal smoothing in the frequency domain removed drifts and improved the signal-to-noise ratio. Functional and anatomical datasets for each subject were aligned and fitted to standardized Talairach space. In this process, the data were resampled at a $3^{\star} 3^{\star} 3 \mathrm{~mm}$ resolution using a trilinear interpolation method, from the initial $33^{\star} 4.5$.

Spectral analysis (Fig. 1)

Spectral analyses were conducted with an inhouse program using MATLAB (The MathWorks). Before the analysis, the data from the periodic experiments were temporally smoothed (Gaussian kernel of $4 \mathrm{~s}$ FWHM) and detrended to remove the mean value and linear drift. Then, following standard retinotopy procedures (Engel et al., 1994; Engel et al., 1997), we applied Fourier analysis to the time course of each voxel, locked to the stimulus repetition frequency. The details of this analysis and the adaptation for use in Brain Voyager and MATLAB programs have been described elsewhere (Hertz and Amedi, 2010; Zeharia et al., 2012). Briefly, the Fourier analysis delivered amplitude and phase values of the time course in each voxel. A pure cosine function was made up of the amplitude and the phase values at the repetition frequency. This cosine profile served as a model of the activation for further statistical evaluation of the results. A Pearson correlation coefficient was calculated between this model's cosine and the time course in each voxel and was used as a direct measure of the voxel's response to the movement (regardless of the specific body part represented in this voxel). The phase value was inspected in regions showing high correlations with the stimulus repetition frequency (threshold at $r>$ 0.36 ). Phase values were distributed between $-\pi$ and $\pi$ and represented time points within each movement cycle. Because the movement of any given body part was always made at a$$
\leftarrow
$$

unsmoothed (US) data. $\boldsymbol{E}$, ROI analysis of data derived from even trials in ROls defined from odd trials confirms the somatotopic preference for leg, hand, and face body parts in different areas of the gradient. $\boldsymbol{F}$, Spectral analysis results confirm the existence of a whole-body somatosensory homunculus in the precuneus, which extends up to the POS and possibly beyond it, in an anterior-posterior, dorsal-ventral orientation. H, Homunculus. 


\section{A Statistical parametric mapping - Event Related - 3 Body Part Groups}
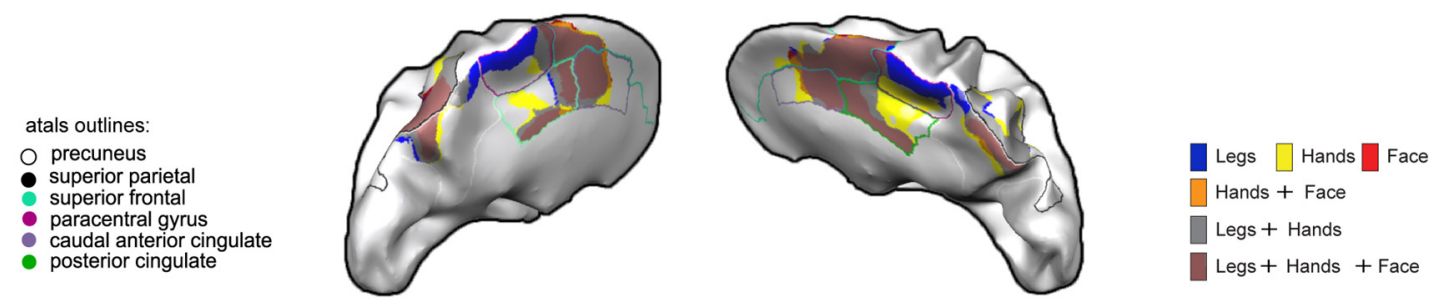

B Peak activation in the gradient - Event Related - 3 Body Part Groups

Anterior
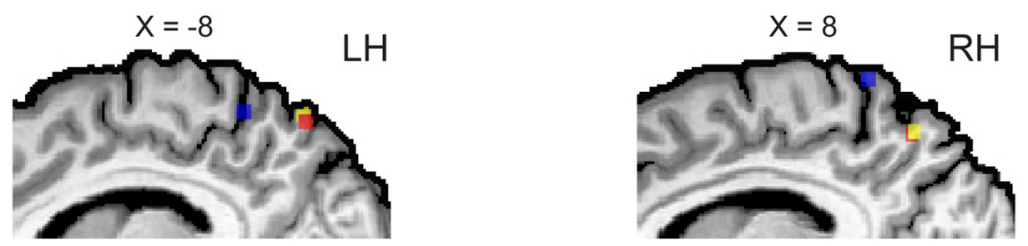

Posterior

\section{Spectral analysis maps in relation to previous reaching-related studies}
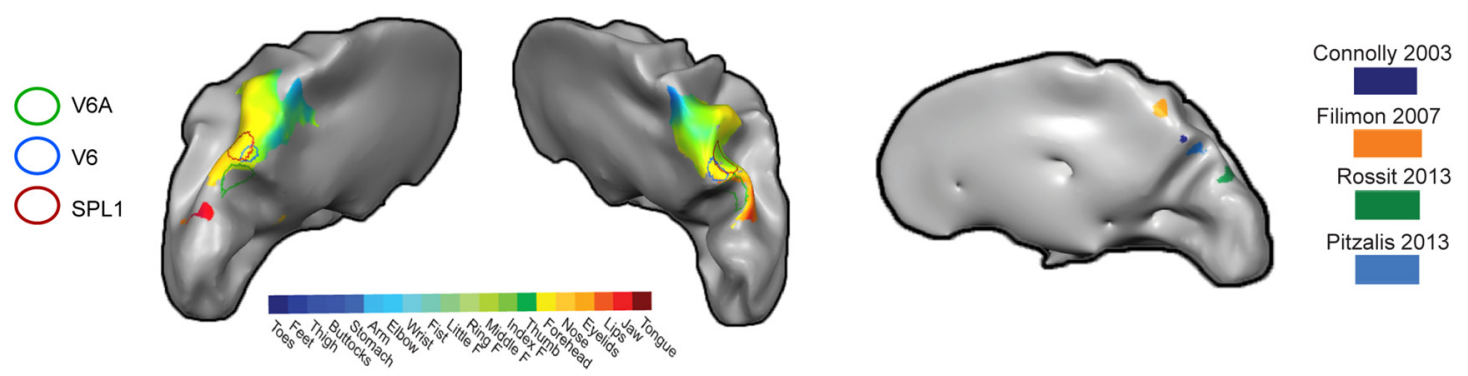

Figure 2. GLM analysis of a randomized event-related experiment confirm the existence of a somatotopic gradient in the precuneus. A, Group random effects (RFX) corrected for multiplecomparisons statistical parametric maps of activations for face (red), hand (yellow), and leg (blue) body parts from a randomized event-related experiment show activation for each of these groups in the precuneus, with substantial overlap. The anatomical landmarks displayed on the surface were used to mask the statistical parametric maps (DKT classifier atlas; Desikan et al., 2006). $\boldsymbol{B}$, Locations of peak activations of the three maps shown in $A$, in the precuneus in each hemisphere. C, Left, Areas V6, V6A, and SPL1 are superimposed on the spectral maps of the posterior homunculi (the anatomical boundaries of these spectral maps are shown in Fig. 1B, right). The anatomical locations of these areas were defined using a $5 \mathrm{~mm}^{3}$ sphere around the coordinates defined in the studies of Pitzalis et al. (2006) (V6), Pitzalis et al. (2013) (V6A), and Konen and Kastner (2008) (SPL1). The averaged phase values in V6 and SPL1 correspond to hands movement. Only a few voxels of the phase maps were found within area V6A. In the right hemisphere, their mean values correspond to face movement. Right, Location of peak neural activation from previous reaching-related studies.

fixed time point in the movement cycle, the phase value served as a measure of the body part being moved. However, because of the time delay of the hemodynamic response, the phase code does not temporally overlap with the movement time (i.e., the phase values correspond to the time of stimulation plus a hemodynamic delay). Furthermore, averaging the gradients of different subjects, which may be slightly shifted relative to one another, may also cause a narrowing of the phase values in the group results.

To control for order effects such as attention at the beginning of the movement cycle, we averaged the results of the "toes to tongue" and "tongue to toes" experiments. The phase values of the "tongue to toes" experiment were flipped, and afterward the phase values of both directions were averaged.

Single-subject results were analyzed both in spatially smoothed (Gaussian kernel of $4 \mathrm{~mm}$ FWHM) and in unsmoothed data, to ensure that the somatotopic gradient was not a result of spatial smoothing of the signal. The group results were compiled using random-effect analysis and corrected for multiple comparisons.

Further details regarding spectral analysis have been described previously (Zeharia et al., 2012).

Analysis of mean phase values along the medial-parietal maps

The group's phase maps were masked to the anatomical boundaries of the precuneus and the superior parietal lobule using the DKT classifier atlas (Desikan et al., 2006). Two lines were drawn on the anterior and posterior borders of the masked maps (Fig. 1C). The phase maps and borderlines were transformed from the surface view to their corresponding voxels in volume space for further analysis. For each voxel in the phase maps (left and right) we extracted a normalized distance along the anterior-posterior cortical surface. This distance was calculated by the ratio of each point in the map to the nearest point on each of the border lines. The normalized distance was then multiplied by the mean length of the map in the anterior-posterior direction, giving a measure of distance along the map for each voxel. Finally, the anterior-posterior direction of the map was divided into bins of $2 \mathrm{~mm}$. Within each bin, we calculated the averaged phase values and their standard error (Harvey et al., 2015).

\section{Regions of interest (ROI) analysis (Fig. 1)}

Three ROIs were defined from the periodic experiments using general linear model (GLM) analysis (rather than spectral analysis). The ROIs were defined across both directions ("toes to tongue" and "tongue to toes"), to avoid the bias of order effects, but only on the odd trials (using the odd movement cycles). The ROIs included the peak activations in both hemispheres of toes, feet and versus rest (legs ROI), all hand body parts versus rest (hands ROI), and tongue, jaw and lips versus rest (face ROI). All the voxels in each ROI were significant $(p<0.05)$ and the size of each ROI was $5^{\star} 5^{\star} 5$ voxels.

GLM analysis was performed in each ROI yielding the GLM parameter estimators of each of the three body parts groups, this time from the even 

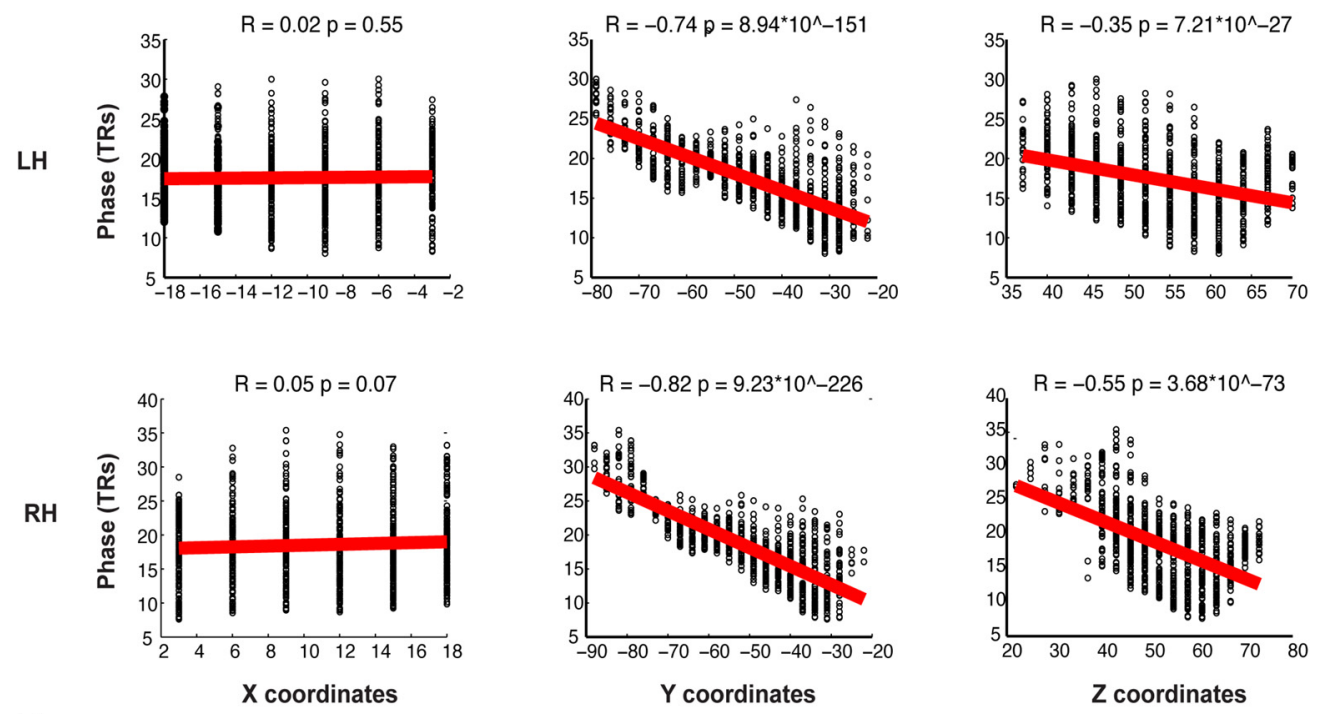

B Linear regression - Group $\mathrm{R}$ values based on group smoothed and singles unsmoothed phase values
Group smoothed phase values

LH RH
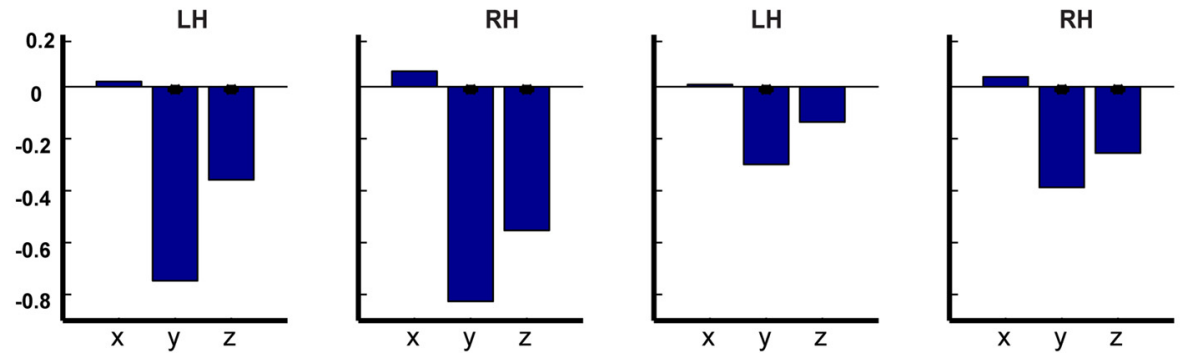

Figure 3. Linear regression confirms the anterior-to-posterior, dorsal-to-ventral, toes-to-tongue directionality of the gradient in the precuneus. $A$, Rsults of linear regression for phase values with respect to the $x$-coordinates (medial-lateral axis), $y$ coordinates (anterior-posterior axis), and $z$ coordinates (dorsal-ventral axis) show that there is a linear change of the phase values along the $y$ and $z$ axes, with a negative correlation coefficient, but not along the $x$-axes. This is a quantitative measure for the anterior-to-posterior, dorsal-to-ventral, toes-to-tongue directionality of the gradient in the precuneus. $\boldsymbol{B}$, Comparison of the correlation coefficients of the linear regression based on group, smoothed phase values (as in $\boldsymbol{A}$ ) compared with the correlation coefficients based on single subjects, unsmoothed phase values, reveals a similar trend.

trials (using the even movement cycles), to avoid "voxel hunting." In addition, the target group was contrasted with the other two (for example, in the legs ROI which was defined from the odd trials, GLM analysis was run on the even trials, and the even legs condition were contrasted with the even hands and even face conditions).

\section{Whole-brain GLM analysis (Fig. 2)}

To further confirm the somatotopy derived from the periodic experimental design and spectral analysis, we performed whole-brain GLM analysis based on the event-related experiment. We contrasted groups of body parts (legs, hands, and face) versus rest (Fig. 2A). All the results presented are random-effect results, corrected for multiple comparisons using the Monte Carlo (Forman et al., 1995) method (1000 iterations, $\alpha<0.05)$. In the ROIs derived from the peak of these maps, all the voxels were significant $(p<0.05)$.

Linear regression analysis (Fig. 3)

To assess the anatomical axes along which the phase gradient was found in the medial wall of the parietal lobe, as well as the directionality of the gradient along each axis, we performed a linear regression analysis using the phase value as the dependent variable and the $\mathrm{x}, y$ or $z$ Talairach coordinates as the independent variable. The phase values were derived either as the group phase values (after spatial smoothing), or as the single subject phase values (without any spatial smoothing). In the latter case, linear regression was carried out separately for each subject, and the correlation coefficients were then averaged to obtain the group correla- tion coefficient. The analysis was conducted separately for the right (900 voxels) and left (904 voxels) hemispheres. The ROI in which the analysis was performed was restricted anatomically to the medial parietal lobe (medial portion of Brodmann area 7), and functionally to voxels with significant response to movement, as found using spectral analysis.

For each axis the significance of the correlation was assessed. A significant correlation along the $x$-axis, $y$-axis, and $z$-axis indicates a significant gradient along the medial-lateral, posterior-anterior and dorsal-ventral axes, respectively. The sign of the correlation coefficient indicates the direction of the gradient (e.g., a positive correlation value in the $y$-axis indicates a posterior (lower $y$ values) to anterior (higher $y$ values), toes (lower phase values) to tongue (higher phase values) gradient, while a negative correlation value indicates an anterior-to-posterior, toes-totongue gradient).

\section{Functional connectivity data analysis and MRI acquisition}

(Figs. 4, 5)

Forty-six participants who were not involved in the periodic experiments took part in this experiment. A dataset of spontaneous BOLD fluctuations for the investigation of intrinsic (rest state; (Biswal et al., 1995) functional connectivity was collected while the subjects lay supine in the scanner without any external stimulation or task. In 33 of the subjects, the data in-plane matrix size was $64 \times 64$, FOV $24 \times 24 \mathrm{~cm}$, TR $=3000$ $\mathrm{ms}$, flip angle $=90^{\circ}$, and TE $=30 \mathrm{~ms}$. In 13 of the subjects, the TR $=2000$ 
LH Seed

A Connectivity map of middle phase from legs and trunk phase values
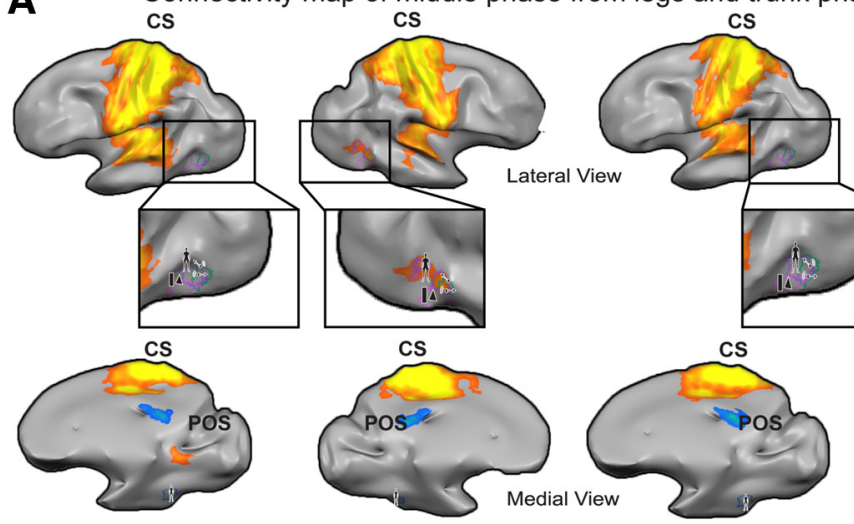

B
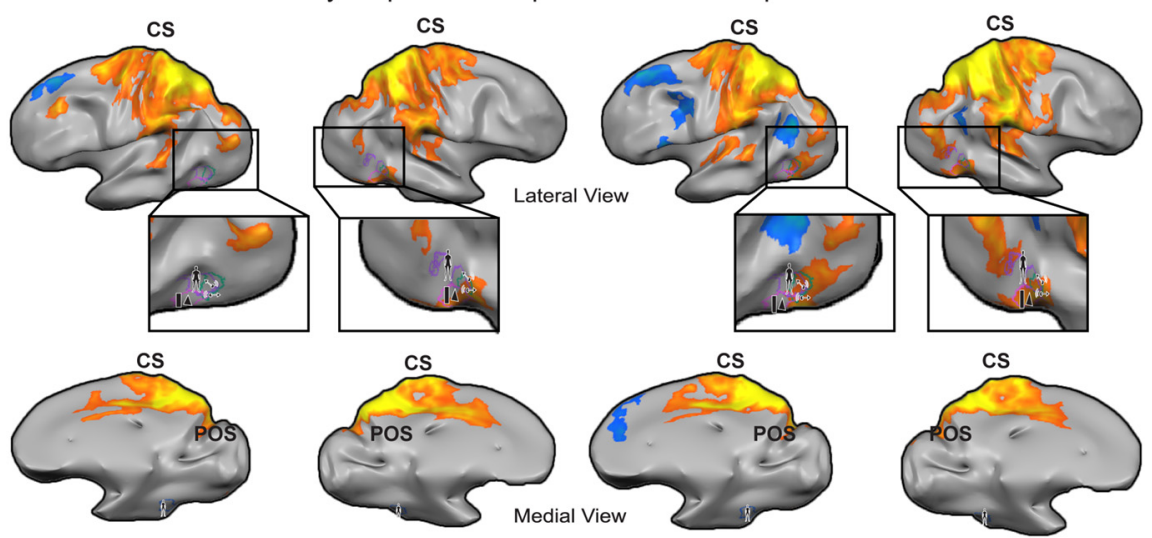

C
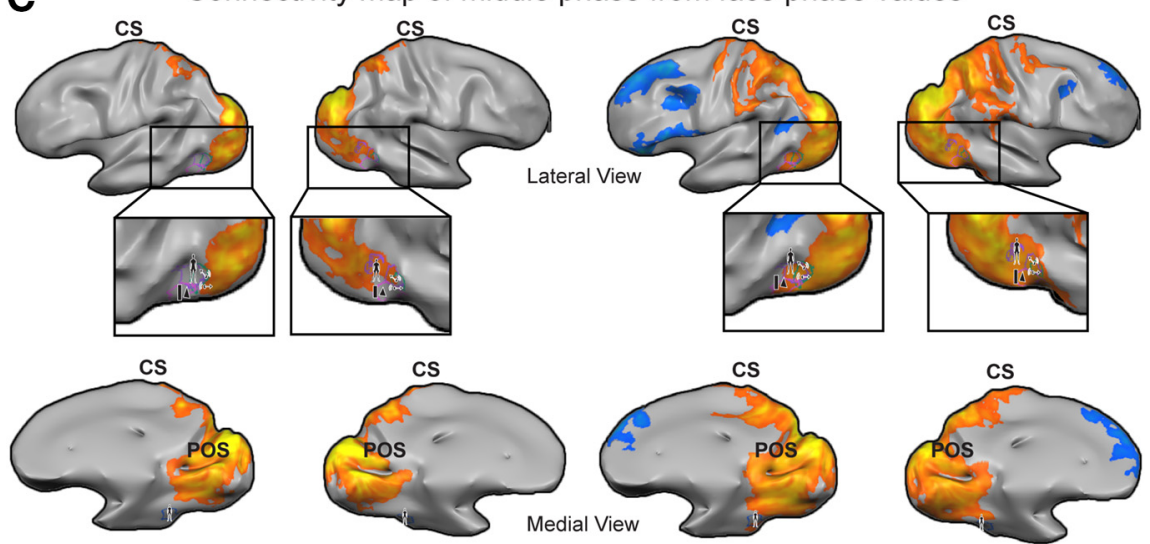

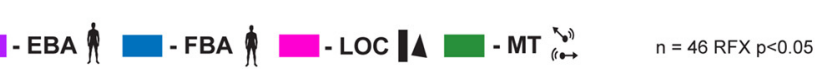

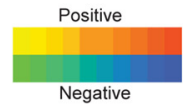

Figure 4. Functional connectivity reveals body-part specific whole-brain connectivity in the precuneus-middle phase examples. $A$, Group ( $n=46$ ) random effects (RFX) corrected for multiple comparisons, functional connectivity results. Functional connectivity results from the seed ROl in the precuneus containing voxels with middle phase values for the leg and trunk-related phase values. Left, Seed derived from the left hemisphere. Right, Seed derived from the right hemisphere. Functional connectivity is found to $M 1 / S 1$, the SMA, the insula, and the EBA. $B$, Functional connectivity results from the seed ROI in the precuneus containing voxels with the middle phase values for the hand-related phase values. Functional connectivity is found to the superior and inferior parietal lobes, the premotor cortex, the caudal cingulate zone, the POS, the frontal lobe, and the EBA. C, Functional connectivity results from the seed $\mathrm{ROI}$ in the precuneus containing voxels with the middle phase value for the face-related phase values. Left, Seed derived from the left hemisphere. Functional connectivity is found to the occipital lobe, the EBA, and the LO complex. The category-related activation in EBA and FBA, LO, and MT were obtained from Talairach coordinates reported in the studies of Peelen and Downing (2005), Amedi et al. (2001), and Rees et al. (2000), respectively. ms. 29-46 slices of $4 \mathrm{~mm}$ thickness were used to obtain full coverage of the subjects' brains.

The number of whole-brain images in each functional scan varied, and were all cropped to 180 images, but the images of the 13 subjects with $\mathrm{TR}=2000$, which were cropped to 158 images. The first two images of each scan were excluded from the analysis because of nonsteady-state magnetization. The ventricles and white matter signals were sampled using a grow-region function embedded in the Brain Voyager from a seed in each individual brain. Using MATLAB, ventricles and white matter time courses were regressed out of the data and the resulting time course was filtered to a frequency bandwidth of $0.1-0.01 \mathrm{~Hz}$ (in which typical spontaneous BOLD fluctuations occur). The data were not spatially smoothed to avoid 'leakage' of information from adjacent voxels.

Seed ROIs were then defined from the same region described in the linear regression in each hemisphere separately. Within this area, the phase values derived from the group spectral analysis of the periodic experiments were rounded off. The seeds were then defined as voxels within this region with a particular phase value. For example, one seed was defined as all the voxels with phase values of eight within the activated medial parietal lobe in the left hemisphere. The range of the rounded off phase values (and thus, of the seed ROIs) was $8-34$ in the left hemisphere and 8-33 in the right hemisphere, resulting in 27 seed ROIs in the left hemisphere and 26 seed ROIs in the right hemisphere.

Assuming linear relationships between the phase values and the movements of the corresponding body parts within the movement cycle, we could approximately assign different phase values to different groups of body parts. The 20 body part movement cycle contained 5 leg and trunk body parts (toes, feet, thighs, buttocks and stomach), 9 hand body parts (upper arm, elbow, wrist, fist, and all 5 fingers), and 6 face body parts (forehead, nose, eyelids, lips, jaw, and tongue). We therefore assigned the first 5/20 phase values ( 7 and 6 first phase values, in the left and right hemisphere, respectively) to the leg and trunk body parts, the next $9 / 20$ phase values (12 phase values) to the hand body parts and the last $6 / 20$ phase values ( 8 phase values) to the face body parts.

After defining the seed ROIs, whole-brain connectivity analysis was performed. Individual time courses of rest data from the separate group of 46 subjects were sampled from these seed ROIs, $z$-normalized and used as individual predictors in a separate group analysis using GLM in a hierarchical random-effects analysis.

This analysis resulted in a separate group map for each one of the seed ROIs. All the maps were false discovery rate (FDR) corrected $(q<$ 0.05).

Category-related localization of the extrastriate body area (EBA), fusiform body area (FBA), lateral occipital (LO), and middle tem- 
poral (MT) were obtained from Talairach coordinates reported in the studies of Peelen and Downing (2005), Amedi et al. (2001), and Rees et al. (2000), respectively. ROIs were built around the reported coordinates of the (256 voxels each). Connectivity analysis was also performed between the seed ROIs of the three body parts and the EBA and MT ROIs. For each subject, correlation tests were conducted between the two functional-category ROIs and each of the different body parts. A $t$ test was then performed on the $\mathrm{r}$ scores of the subjects, followed by FDR correction for multiple comparisons.

Finally, a probability map was calculated for each set of maps derived from the seed ROIs assigned to the same group of body parts. For example, all the maps of the first seven seed ROIs in the left hemisphere, which were assigned as leg seed ROIs, served to build one probability map, in which the value in each voxel was the percentage of maps (out of these seven maps) with significant activation in this voxel. This was done separately for the leg and trunk, hand and face maps, and separately for the right and left hemisphere.

\section{Results}

In their 1954 study, Penfield and Jasper predicted the existence of a "supplementary sensory" homunculus in the medial wall of the parietal lobe, mirrored to the SMA (Penfield and Jasper, 1954) (Fig. $1 A)$. This study examined the medial wall of the parietal lobe using a periodic experimental design and spectral analysis (see Materials and Methods) to determine whether evidence for a whole-body, sensory-motor gradient would be found in this area.

During the periodic experiments (see Materials and Methods), subjects were asked to move 20 body parts separately and consecutively in a fixed order, from "toes to tongue" and from "tongue to toes." All movements were synchronized in-phase bilateral movements. The continuous cyclic design of these experiments allowed us to analyze the BOLD signal in every voxel using spectral analysis (Engel et al., 1994; Engel et al., 1997; Sereno and Huang, 2006; Hertz and Amedi, 2010; Engel, 2012; Besle et al., 2013) (see Materials and Methods).

The group results of spectral analysis (Fig. $1 B$ ) show a whole-body sensory-motor gradient in the medial wall of the parietal lobe (gradient "1") in both hemispheres, somewhat more lateral from the midline (Fig. $1 D, x= \pm 8$ and $x= \pm 14$ ). This gradient seems to progress in an anterior-toposterior, dorsal-to-ventral, toes-to-tongue orientation. The SMA (gradient " 2 ") lies in a mirror orientation to this gradient, with the legs area of M1 between these two areas. (2000), respectively.

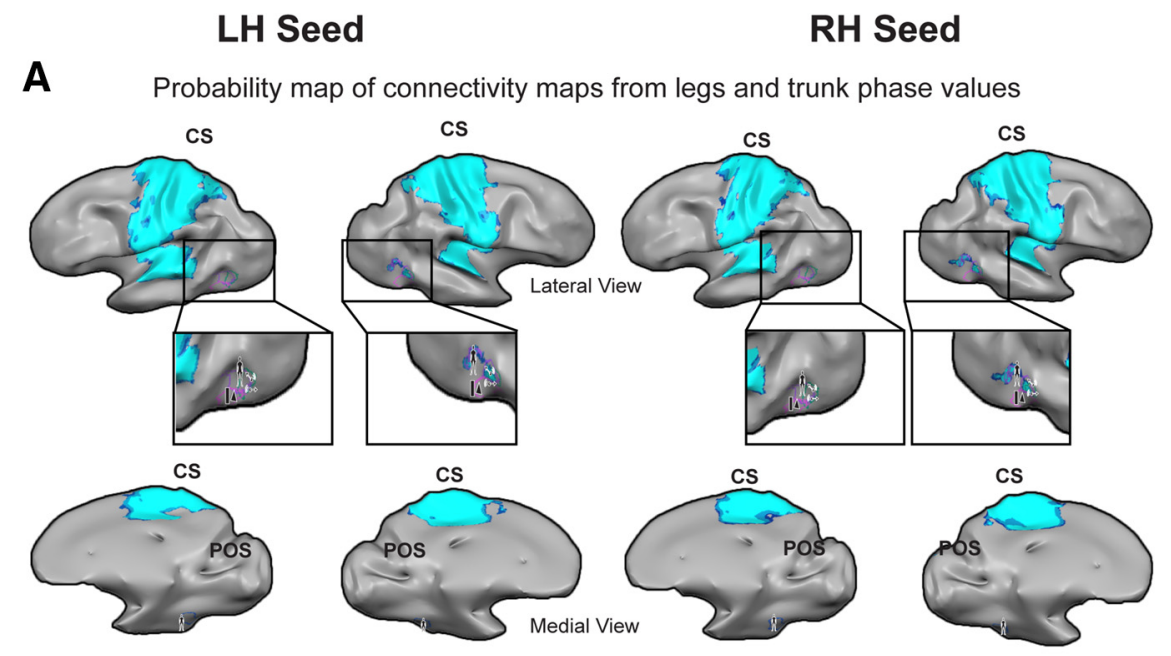

B Probability map of connectivity maps from hands phase values

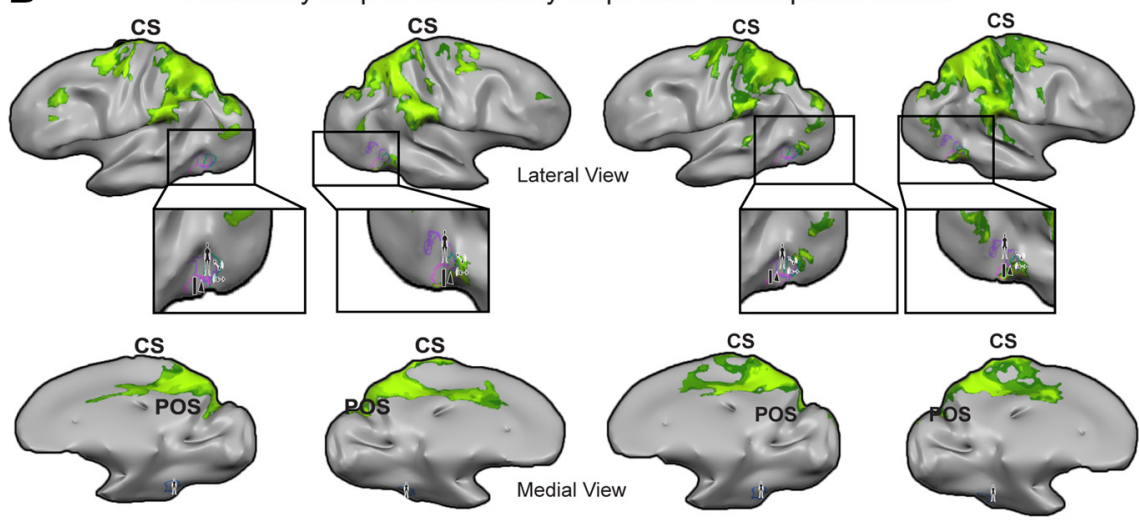

C Probability map of connectivity maps from face phase values

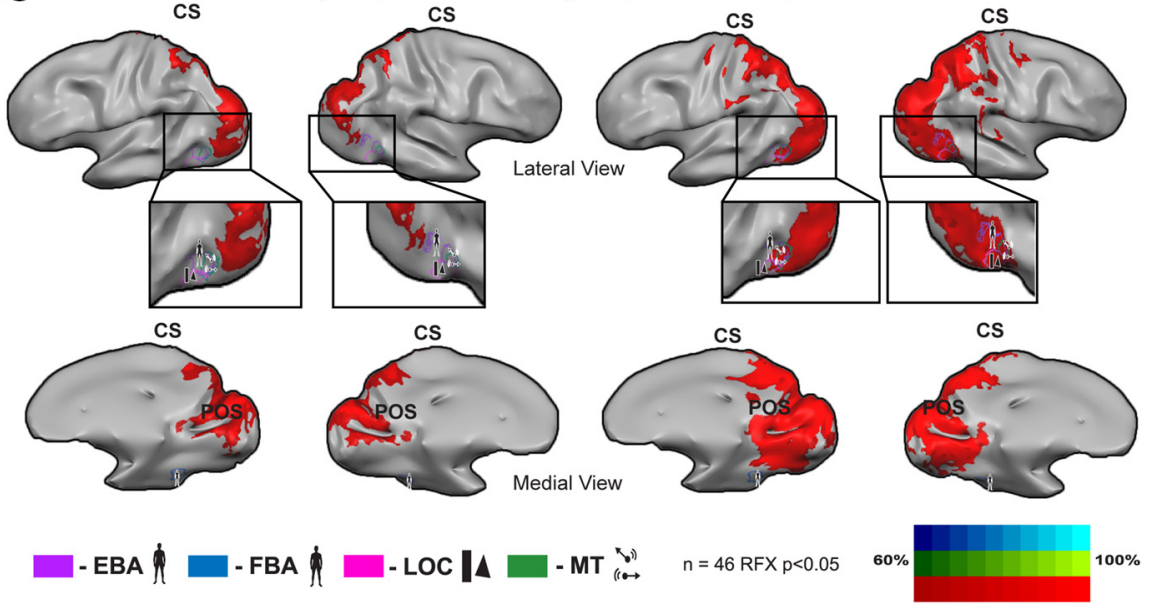

Figure 5. Functional connectivity reveals body-part-specific whole-brain connectivity in the precuneus probability maps. Group $(n=46)$ random effects (RFX) corrected for multiple comparisons, functional connectivity results. Same as in Figure 4, but instead of a representative functional connectivity map for each group of body parts, a probability map is presented for all the functional connectivity maps for the leg and trunk $(\boldsymbol{A})$, hand $(\boldsymbol{B})$, and face $(\boldsymbol{C})$ seed ROls in each hemisphere. The value of each voxel is the percentage of significantly activated maps for the functional connectivity maps of the relevant group of body parts in this voxel. The category-related activation in EBA and FBA, LO, and MT were obtained from Talariach coordinates reported in the studies of Peelen and Downing (2005), Amedi et al. (2001), and Rees et al.

The gradient is also visible when inspecting the data of single subjects, both with and without spatial smoothing (Fig. 1D).

ROI analysis (Fig. 1E), in which peak activation for legs, hands and face in both hemispheres were defined from the odd trials 
and data from the even trials were analyzed (see Materials and Methods) also support the representation of different parts of the body in this gradient. This is evident both when inspecting the GLM parameter estimators of each group of body parts in each ROI, and when contrasting each group to the two others (for example, when contrasting the even legs condition to the even hands and face conditions in the odd legs ROI). From the ROI analysis it is evident that the face representation in this gradient is weaker compared with the hand and leg representation.

These findings can be applied to revise model by Penfield and Jasper (1954) (Fig. 1F) and suggest that there indeed is a wholebody sensory or motor gradient at the medial wall of the parietal lobe. Furthermore, this gradient might contain more body parts and proceed more posteriorly than suggested by Penfield and Jasper (1954), possibly even crossing the POS and reaching the anterior edge of the occipital lobe.

Although spectral analysis is highly sensitive to the detection of gradients of representations across brain areas as well as subtle preferences within voxels (Engel, 2012), GLM analysis of randomized and segregated blocks of movements may yield more accurate information regarding the exact identity of the represented body parts in each voxel and the possible overlap in the representation of different body parts.

We therefore analyzed an event-related experiment, in which the subjects made the exact same movements with the same 20 body parts, only in a randomized manner, with a rest period between the movements of different body parts (see Materials and Methods). We first divided the 20 body parts into legs (blue), hands (yellow), and face (red) body parts (Fig. 2A). Using a permissive, yet statistically significant threshold, GLM analysis on these groups revealed that each activated the area of the gradient found by spectral analysis in the precuneus; suggesting that this area indeed represents leg, hand and face movements or sensation. The activation of the legs was located in the most anterior part of the precuneus, continuous with the legs representation of the SMA and M1. The activations of the hands and face stretched from the most anterior to the most posterior part of the precuneus. GLM analysis also revealed a substantial overlap in the representation of the different body parts, which is typical in sensory-motor gradients, and cannot be identified with spectral analysis.

When inspecting the peak of representation of each group of body parts (Fig. $2 B$ ) in the precuneus, the peak representation of legs emerged as more anterior, in accordance to the gradient found in spectral analysis, whereas the peaks of hands and face were more anterior and overlapped.

To further quantitatively assess the existence and directionality of the somatotopic gradient in this area, we used all the voxels identified by spectral analysis to be part of this gradient for an additional linear regression analysis (Fig. 3).

Initially, we ran the linear regression by setting the group phase values (which are related to the body part that moved) as the dependent variable and the $\times$ Talairach coordinates as the independent variable. The $\times$ Talairach coordinates change along the medial-lateral axis. Therefore, a linear change of the phase values, that is, a gradient-along the $x$-axis, would be suggestive of a somatotopic gradient in the medial-lateral axis. However, no such gradient was found, either in the left or right hemisphere (Fig. 3A, left column).

In contrast, there were clear linear relationships in both hemispheres between the $y$ Talairach coordinates-which change along the posterior-anterior axis - and the phase values, with a negative correlation coefficient (Fig. $3 A$, middle column). Thus, there is a gradient along this axis. The sign of the correlation coefficient shows that as the $y$ coordinates increase, the phase values decrease, suggesting that the directionality of the gradient is anterior-to-posterior, toes-to-tongue.

Similarly, a dorsal-to-ventral, toes-to-tongue gradient was identified in both hemispheres from the results of the linear regression using the $z$ Talairach coordinates as the independent variable (Fig. $3 A$, right column). This gradient had a lower correlation coefficient than the anterior-to-posterior gradient, but was still highly significant.

In addition, although the slope of the gradient along the mediallateral ( $x$-axis) direction was very low ( 0.01 and 0.06 for the left and right hemispheres, respectively), the slope along the anterior-posterior ( $y$-axis) direction was much higher $(-0.21$ and -0.27 for the left and right hemispheres, respectively), and similar to the slope along the dorsal-ventral ( $z$-axis) direction $(-0.18$ and -0.28 for the left and right hemispheres, respectively).

To confirm that the results were not an artifact of the spatial smoothing or the averaging of the phase values of single subjects, we ran the same linear regression analysis for each subject separately, without any spatial smoothing of the phase values. We then averaged the correlation coefficients of all the single subjects separately for each axis and hemisphere.

When comparing the correlation coefficients derived when using the group, spatially smoothed results (Fig. 3B, left graphs) to the correlation coefficients derived when using the single subject, non-spatially smoothed results (Fig. 3B, right graphs), the trend was still highly similar although the correlation coefficient values for the single subjects were lower. There were no linear relationships between the phase values and the $x$-axis (i.e., no somatotopic gradient in the medial-lateral direction), but there was quantitative, significant evidence for an anterior-to-posterior, toes-to-tongue gradient along the $y$-axis. Similar to the group results, in the single subject analyses the correlation coefficients of the linear regression were higher when using the $y$-axis as the independent variable than when using the $z$-axis. Finally, the dorsal-to-ventral, toes-to-tongue gradient along the $z$-axis was only significant in the right hemisphere. In the left hemisphere the same trend was observed, but it was not significant.

Overall, both the group and single subject analysis yielded very similar results: a quantitative evidence for an anterior-toposterior, dorsal-to-ventral, toes-to-tongue gradient in the medial wall of the parietal lobe and the anterior edge of the occipital lobe.

To gain further insights into the functional role of the gradient and specifically the roles of the represented body parts in this gradient, we performed a whole-brain functional connectivity analysis using rest data from a separate group of subjects (see Materials and Methods). We aimed at defining the seed ROIs within this gradient based on the different represented body parts. The gradient was therefore divided into 27 and 26 seed ROIs in the left and right hemisphere, respectively. The gradients were defined based on the rounded-off phase values, that is, all the voxels in the gradient with the same rounded-off phase value and in the same hemisphere were defined as one seed ROI. Assuming linearity between the phase values and the moving body parts, each phase value (and therefore each seed ROI) could be approximately attributed to either leg and trunk, hand or face body parts. Figure 4 presents the connectivity map of the middle seed ROI of each approximated body part group (i.e., if the first seven maps are approximately defined as leg and trunk maps, the fourth one is presented in Fig. 4A). When using voxels with low 
phase values-corresponding to leg body parts-as seeds, connections were found mainly to M1/S1, both on the lateral and medial wall, the SMA, the insula, MT (middle temporal), and the right EBA (extrastriate body area). The connectivity pattern was similar, both when choosing the ROIs from the left hemisphere and from the right hemisphere.

When inspecting the connectivity patterns of seeds ROIs with middle phase values-corresponding to hand body parts-as seeds (Fig. 4B), connections were found mainly to the parietal lobe, both medial and lateral (in the superior and inferior areas), the POS, the cingulate sulcus, the premotor cortex, the frontal cortex, MT and the EBA. These connections were also similar, regardless of the hemisphere from which the seed ROIs were extracted. However, the connectivity with the EBA and MT was weaker when the left hand phase values were used as seeds.

Connections to seed ROIs defined by later phase values, corresponding to face body parts, were found mainly to the bilateral occipital lobe, as well as to MT, EBA, and the LO complex (Fig. $4 C)$. The connectivity to the MT, EBA, and LO was, however, weaker when the left face values were used as seeds.

When inspecting the correlation across subjects between the left and right EBA and MT and the different body parts, most correlations were found significant ( $p<0.05$, FDR corrected). The correlations that were not found to be significant were between the right EBA and the left hands and face seeds $(p=0.051$ and 0.12 , respectively), the left EBA and the right legs seed $(p=$ $0.15)$ and the left MT with the right legs seed ( $p=0.058)$. A two-way ANOVA analysis between the three body parts segments (face, hands, legs) and the functional ROIs (EBA, MT) failed to find any significant differences in the correlation values between the different ROIs or body parts.

The same pattern of connectivity can be seen when looking at the probability maps of the three groups of body parts (Fig. 5). In these maps, e.g., the connectivity maps of all leg and trunk related seed ROIs ( 7 and 6 maps in the left and right hemisphere, respectively) served to build one probability map (Fig. $5 \mathrm{~A}$ ), in which the value of each voxel was the percentage of these maps which were significant. The same was done for the 12 hand related connectivity maps (Fig. $5 B$ ) and the eight face-related connectivity maps (Fig. 5C). These maps further confirm the body-part specific, bilateral connectivity pattern depicted in Figure 4.

Overall, the phase-specific connectivity analysis of the gradient shows a shift from connectivity mainly to motor areas (using low phase seeds), through connectivity to mainly to parietal and secondary motor areas (using middle phase seeds), to connectivity to occipital areas (using high phase seeds), whereas all areas were also connected to MT and the EBA (mainly in the right hemisphere).

\section{Discussion}

Sensory and motor representations have been found in the parietal lobe, outside S1, both on the lateral and the medial surfaces. On the lateral surface, multisensory and motor functionality have been reported (Bremmer et al., 2001; Culham and Valyear, 2006; Huang et al., 2012; Sereno and Huang, 2014) and multiple retinotopic maps, as well as a congruent whole-body somatosensory map have been identified (Sereno and Huang, 2006; Huang et al., 2012; for review, see Sereno and Huang, 2014), along with motorrelated activations, mainly for grasping, but also for reaching (Hagler et al., 2007; Filimon et al., 2009; Cavina-Pratesi et al., 2010; Vesia et al., 2010; Konen et al., 2013).

Penfield and Jasper (1954) also hypothesized the existence of a whole-body sensory representation in the medial part of the pa- rietal lobe, but this has never been proved. In recent years, evidence has accumulated for motor-related activations, but their exact location and functionality are not clear.

Konen et al. (2013) reported evidence for a grasping-toreaching, anterior lateral-to-posterior medial gradient at the parietal lobe. In the posterior part of the precuneus (Pitzalis et al., 2013), a human homolog of macaque area V6A, in which both retinotopic and reaching-related activations were found, was located near or in the anterior bank of the POS, an area which is located at the posterior edge of the medial parietal lobe, in contrast to solely visual activations in the posterior bank of the same sulcus, which is located at the anterior edge of the occipital lobe. In contrast, other studies have shown that hand reaching-related activations can be found both anterior and posterior to the POS (Connolly et al., 2003; Cavina-Pratesi et al., 2010), implying that these activations are continuous across the medial wall of the parietal and occipital lobe. Evidence for reaching-related functionality of the POS have also been documented using TMS (Vesia et al., 2010).

Other studies have also found reaching-related activations at the medial wall of the parietal lobe, both at the POS but also in more anterior positions (Connolly et al., 2003; Filimon et al., 2007; Rossit et al., 2013). These more anterior positions can be attributed to a proprioceptive-motor reaching-related activation, whereas the POS activation can be related to visuomotor tasks (Filimon et al., 2007); both activations in both regions were related to lower rather than to higher visual field reaching (Rossit et al., 2013). It was suggested that the precuneus is part of a reaching mirror system (Filimon et al., 2007), activated by executing, observing, and imagination of reaching.

Consistent with the results presented, we show using GLM analysis (Fig. 2A) that hand-related activations spread across the entire precuneus, from the marginal ramus of the cingulate sulcus anteriorly to the POS posteriorly. However, activations in response to leg and face movements were also evident, and the sensitivity of spectral analysis (Fig. $1 B, C$ ) indicated a shift in the preference of the voxels from toes-to-tongue along these two edges.

Therefore, along with the clear role of the precuneus in reaching movements, visually guided hand movements, and imagery of movements, it also represents other body parts, specifically, a whole-body, overlapping gradient.

\section{Functional connectivity of the gradient}

Margulies et al. (2009) subdivided the precuneus into three parts based on functional connectivity results, and suggested that the anterior part is a sensory-motor area connected to the motor cortex, the insula, and the superior parietal lobe; the middle part is a cognitive-associative area connected to the prefrontal lobe and the inferior parietal lobe; and the posterior part is a visualrelated area connected to visual areas in the occipital lobe.

Here, seed ROIs from the precuneus were defined based on functional preference to legs, hands, or face rather than anatomical markers. However, the two divisions share some similarities as most of the leg-responsive voxels were located in the anterior portion of the gradient, most of the face-responsive voxels were located in the posterior portion of the gradient (in both banks of the POS), and the hand-responsive voxels were located between them.

The functional connectivity results confirm and are similar to the results in Margulies et al. (2009), but with several important differences. The leg-related voxels, which are the most anterior voxels, were found to be functionally connected to motor areas such as M1/S1 and the SMA, but also to the visual EBA area, 
which is activated when visually observing body parts and to area MT. The hand-related voxels were found to be functionally connected not only to parietal areas, including the superior and inferior parietal lobes, but also to secondary motor areas such as the caudal cingulate zone and the premotor cortex. There was also a connection to MT and the EBA. Finally, face-related voxels were found to be connected to the occipital lobe, including the high visual areas, but also late visual areas such as MT, EBA, and LO.

The novel connections to the EBA from all body parts may suggest that the whole-body topography we found in the precuneus is functionally connected to body-parts observations. This is consistent with the role of the precuneus in reaching observation (Filimon et al., 2007). Future research should investigate whether this functional connectivity is somatotopic, body-part specific because both the precuneus and the EBA (Orlov et al., 2010) seem to be topographically organized.

Both the leg and hand areas of the precuneus are connected to primary and secondary sensory-motor areas, such as M1, the SMA (legs), caudal cingulate zone and the premotor cortex (hands). This suggests that the sensory-motor area is not confined to the anterior portion of the precuneus but might proceed more posteriorly.

The most posterior, face-related connections to the visual system were found in seed ROIs which lie in both banks of the POS. Previous studies have also acknowledged the possibility of crossPOS activations (Connolly et al., 2003; Cavina-Pratesi et al., 2010), although they refer to hand movements. Recent work has identified a human homolog of area V6A which is retinotopic in the anterior bank of the POS and is related to reaching (Pitzalis et al., 2013). Our results (Figs. 1,2) do not rule out the possibility of hand-related activations in this area, but support the fact that both hand- and face-related activations can be found in it, with a preference for face activations. This may imply that this retinotopic area, which is also functionally connected to the visual system, should be treated as a hand-face, visually connected area.

\section{Confounding factors and future directions}

The gradient that we have found is a somatotopic gradient; that is, it is body related. However, given the current experimental design, it is impossible to distinguish whether it is a sensory (touch or proprioceptive) gradient or a motor gradient. Additional experiments targeting only the sensory components might shed light on this issue. Furthermore, despite the extensive training, EMG recording were taken inside the scanner from only several subjects and from a small subset of muscles. It is therefore possible that some rare mistakes in movement happened inside the scanner in spite the extensive training (but this is quite unlikely to influence our results in a significant way).

Further research is needed to elucidate the specific role of the new precuneus homunculus. Another interesting future direction is the putative links of the insula (Zeharia et al., 2012) and the current precuneus homunculus. These two homunculi suggesting that topographical representations might be located also in areas that underlie higher-level cognitive functions (for review, see Cavanna and Trimble, 2006), two areas that a previous study found to be highly connected (Margulies et al., 2009). It was suggested that mind-body interventions can enhance emotional well-being by reducing the constant train of thoughts and the emotional toll they take by focusing our attention on the body. Mind-body interventions seem to affect both self-referential processes as well as body-related representations, and these may be coupled. The findings on precuneus and insula homunculi might provide a novel putative important node linking body and mind, but future studies must study these questions in a more direct and controlled manner.

\section{References}

Amedi A, Malach R, Hendler T, Peled S, Zohary E (2001) Visuo-haptic object-related activation in the ventral visual pathway. Nat Neurosci 4:324-330.

Besle J, Sánchez-Panchuelo RM, Bowtell R, Francis S, Schluppeck D (2013) Single-subject fMRI mapping at $7 \mathrm{~T}$ of the representation of fingertips in S1: a comparison of event-related and phase-encoding designs. J Neurophysiol 109:2293-2305.

Biswal B, Zerrin Yetkin FZ, Haughton VM, Hyde JS (1995) Functional connectivity in the motor cortex of resting human brain using echo-planar MRI. Magn Reson Med 34:537-541.

Bremmer F, Schlack A, Shah NJ, Zafiris O, Kubischik M, Hoffmann K, Zilles K, Fink GR (2001) Polymodal motion processing in posterior parietal and premotor cortex: a human fMRI study strongly implies equivalencies between humans and monkeys. Neuron 29:287-296.

Cavanna AE, Trimble MR (2006) The precuneus: a review of its functional anatomy and behavioural correlates. Brain 129:564-583.

Cavina-Pratesi C, Monaco S, Fattori P, Galletti C, McAdam TD, Quinlan DJ, Goodale MA, Culham JC (2010) Functional magnetic resonance imaging reveals the neural substrates of arm transport and grip formation in reach-to-grasp actions in humans. J Neurosci 30:10306-10323.

Chainay H, Krainik A, Tanguy ML, Gerardin E, Le Bihan D, Lehéricy S (2004) Foot, face and hand representation in the human supplementary motor area. Neuroreport 15:765-769.

Connolly JD, Andersen RA, Goodale MA (2003) FMRI evidence for a'parietal reach region'in the human brain. Exp Brain Res 153:140-145.

Culham JC, Valyear KF (2006) Human parietal cortex in action. Curr Opin Neurobiol 16:205-212.

Desikan RS, Ségonne F, Fischl B, Quinn BT, Dickerson BC, Blacker D, Buckner RL, Dale AM, Maguire RP, Hyman BT, Albert MS, Killiany RJ (2006) An automated labeling system for subdividing the human cerebral cortex on MRI scans into gyral based regions of interest. Neuroimage 31:968980.

Engel SA (2012) The development and use of phase-encoded functional MRI designs. Neuroimage 62:1195-1200.

Engel SA, Rumelhart DE, Wandell BA, Lee AT, Glover GH, Chichilnisky EJ, Shadlen MN (1994) fMRI of human visual cortex. Nature 369:525.

Engel SA, Glover GH, Wandell BA (1997) Retinotopic organization in human visual cortex and the spatial precision of functional MRI. Cereb Cortex 7:181-192.

Filimon F, Nelson JD, Hagler DJ, Sereno MI (2007) Human cortical representations for reaching: mirror neurons for execution, observation, and imagery. Neuroimage 37:1315-1328.

Filimon F, Nelson JD, Huang RS, Sereno MI (2009) Multiple parietal reach regions in humans: cortical representations for visual and proprioceptive feedback during on-line reaching. J Neurosci 29:2961-2971.

Forman SD, Cohen JD, Fitzgerald M, Eddy WF, Mintun MA, Noll DC (1995) Improved assessment of significant activation in functional magnetic resonance imaging (fMRI): use of a cluster-size threshold. Magn Reson Med 33:636-647.

Hagler DJ Jr, Riecke L, Sereno M (2007) Parietal and superior frontal visuospatial maps activated by pointing and saccades. Neuroimage 35:15621577.

Hanakawa T, Immisch I, Toma K, Dimyan MA, Van Gelderen P, Hallett M (2003) Functional properties of brain areas associated with motor execution and imagery. J Neurophysiol 89:989-1002.

Harvey BM, Fracasso A, Petridou N, Dumoulin SO (2015) Topographic representations of object size and relationships with numerosity reveal generalized quantity processing in human parietal cortex. Proc Natl Acad Sci U S A 112:13525-13530.

Hertz U, Amedi A (2010) Disentangling unisensory and multisensory components in audiovisual integration using a novel multifrequency fMRI spectral analysis. Neuroimage 52:617-632.

Huang RS, Chen CF, Tran AT, Holstein KL, Sereno MI (2012) Mapping multisensory parietal face and body areas in humans. Proc Natl Acad Sci U S A 109:18114-18119.

Konen CS, Kastner S (2008) Representation of eye movements and stimulus motion in topographically organized areas of human posterior parietal cortex. J Neurosci 28:8361-8375. 
Konen CS, Mruczek RE, Montoya JL, Kastner S (2013) Functional organization of human posterior parietal cortex: grasping-and reaching-related activations relative to topographically organized cortex. J Neurophysiol 109:2897-2908.

Malouin F, Richards CL, Jackson PL, Dumas F, Doyon J (2003) Brain activations during motor imagery of locomotor-related tasks: a PET study. Hum Brain Mapp 19:47-62.

Margulies DS, Vincent JL, Kelly C, Lohmann G, Uddin LQ, Biswal BB, Villringer A, Castellanos FX, Milham MP, Petrides M (2009) Precuneus shares intrinsic functional architecture in humans and monkeys. Proc Natl Acad Sci U S A 106:20069-20074.

Meier JD, Aflalo TN, Kastner S, Graziano MS (2008) Complex organization of human primary motor cortex: a high-resolution fMRI study. J Neurophysiol 100:1800-1812.

Orlov T, Makin TR, Zohary E (2010) Topographic representation of the human body in the occipitotemporal cortex. Neuron 68:586-600.

Peelen MV, Downing PE (2005) Selectivity for the human body in the fusiform gyrus. J Neurophysiol 93:603-608.

Penfield W, Boldrey E (1937) Somatic motor and sensory representation in the cerebral cortex of man as studied by electrical stimulation. Brain, pp $389-443$.

Penfield W, Jasper H (1954) Epilepsy and the functional anatomy of the human brain. Oxford, England: Little, Brown \& Co.

Pitzalis S, Galletti C, Huang RS, Patria F, Committeri G, Galati G, Fattori P, Sereno MI (2006) Wide-field retinotopy defines human cortical visual area V6. J Neurosci 26:7962-7973.
Pitzalis S, Sereno MI, Committeri G, Fattori P, Galati G, Tosoni A, Galletti C (2013) The human homologue of macaque area V6A. Neuroimage 82:517-530.

Rees G, Friston K, Koch C (2000) A direct quantitative relationship between the functional properties of human and macaque V5. Nat Neurosci 3:716-723.

Rossit S, McAdam T, McLean DA, Goodale MA, Culham JC (2013) fMRI reveals a lower visual field preference for hand actions in human superior parieto-occipital cortex (SPOC) and precuneus. Cortex 49:2525-2541.

Sereno MI, Huang RS (2006) A human parietal face area contains aligned head-centered visual and tactile maps. Nat Neurosci 9:1337-1343.

Sereno MI, Huang RS (2014) Multisensory maps in parietal cortex. Curr Opin Neurobiol 24:39-46.

Talairach J, Tournoux P (1988) Co-planar stereotaxic atlas of the human brain: 3-dimensional proportional system: an approach to cerebral imaging. New York: Thieme.

Vesia M, Prime SL, Yan X, Sergio LE, Crawford JD (2010) Specificity of human parietal saccade and reach regions during transcranial magnetic stimulation. J Neurosci 30:13053-13065.

Wenderoth N, Debaere F, Sunaert S, Swinnen SP (2005) The role of anterior cingulate cortex and precuneus in the coordination of motor behaviour. Eur J Neurosci 22:235-246.

Zeharia N, Hertz U, Flash T, Amedi A (2012) Negative blood oxygenation level dependent homunculus and somatotopic information in primary motor cortex and supplementary motor area. Proc Natl Acad Sci U S A 109:18565-18570. 